\title{
Transition of Consistency and Satisfiability under Language Extensions ${ }^{1}$
}

\author{
Julian J. Schlöder \\ Mathematisches Institut \\ Rheinische Friedrich-Wilhelms-Universität Bonn \\ Endenicher Allee 60 \\ D-53113 Bonn, Germany \\ Peter Koepke \\ Mathematisches Institut \\ Rheinische Friedrich-Wilhelms-Universität Bonn \\ Endenicher Allee 60 \\ D-53113 Bonn, Germany
}

\begin{abstract}
Summary. This article is the first in a series of two Mizar articles constituting a formal proof of the Gödel Completeness theorem [17] for uncountably large languages. We follow the proof given in [18]. The present article contains the techniques required to expand formal languages. We prove that consistent or satisfiable theories retain these properties under changes to the language they are formulated in.
\end{abstract}

MML identifier: QC_TRANS, version: $\underline{7.14 .014 .183 .1153}$

The notation and terminology used in this paper have been introduced in the following papers: [8], [1], [2], [11], [16], [4], [15], [12], [13], [7], [6], [22], [3], [19], [23], [24], [5], [20], [9], [10], [21], and [14].

\footnotetext{
${ }^{1}$ This article is part of the first author's Bachelor thesis under the supervision of the second author.

(C) 2012 University of Białystok CC-BY-SA License ver. 3.0 or late ISSN $1426-2630(\mathrm{p}), 1898-9934(\mathrm{e})$
} 


\section{LANGuAge Extensions}

For simplicity, we adopt the following rules: $A_{1}$ denotes an alphabet, $P_{1}$ denotes a consistent subset of CQC-WFF $A_{1}, p, r$ denote elements of CQC-WFF $A_{1}$, $A$ denotes a non empty set, $J$ denotes an interpretation of $A_{1}$ and $A, v$ denotes an element of the valuations in $A_{1}$ and $A, k$ denotes a natural number, $l$ denotes a CQC-variable list of $k$ and $A_{1}, P$ denotes a predicate symbol of $k$ and $A_{1}$, and $x, y$ denote bound variables of $A_{1}$.

Let us consider $A_{1}$ and let $A_{2}$ be an alphabet. We say that $A_{2}$ is $A_{1}$ expanding if and only if:

(Def. 1) $A_{1} \subseteq A_{2}$.

Let us consider $A_{1}$. Note that there exists an alphabet which is $A_{1}$-expanding.

Let $A_{3}, A_{4}$ be countable alphabets. One can check that there exists an alphabet which is countable, $A_{3}$-expanding, and $A_{4}$-expanding.

Let $A_{1}, A_{4}$ be alphabets and let $P$ be a subset of CQC-WFF $A_{1}$. We say that $P$ is $A_{4}$-consistent if and only if:

(Def. 2) For every subset $S$ of CQC-WFF $A_{4}$ such that $P=S$ holds $S$ is consistent.

Let us consider $A_{1}$. One can check that there exists a subset of CQC-WFF $A_{1}$ which is non empty and consistent.

Let us consider $A_{1}$. One can check that every subset of CQC-WFF $A_{1}$ which is consistent is also $A_{1}$-consistent and every subset of CQC-WFF $A_{1}$ which is $A_{1}$-consistent is also consistent.

For simplicity, we follow the rules: $A_{4}$ is an $A_{1}$-expanding alphabet, $J_{2}$ is an interpretation of $A_{4}$ and $A, J_{1}$ is an interpretation of $A_{1}$ and $A, v_{2}$ is an element of the valuations in $A_{4}$ and $A$, and $v_{1}$ is an element of the valuations in $A_{1}$ and $A$.

Next we state several propositions:

(1) $\operatorname{Arity}(P)=\operatorname{len} l$.

(2) Symb $A_{1} \subseteq$ Symb $A_{4}$.

(3) The predicate symbols of $A_{1} \subseteq$ the predicate symbols of $A_{4}$.

(4) The bound variables of $A_{1} \subseteq$ the bound variables of $A_{4}$.

(5) For every $k$ holds every $l$ is a CQC-variable list of $k$ and $A_{4}$.

(6) $P$ is a predicate symbol of $k$ and $A_{4}$.

(7) For every $A_{1}$-expanding alphabet $A_{4}$ holds every $p$ is an element of CQC-WFF $A_{4}$.

Let us consider $A_{1}$, let $A_{4}$ be an $A_{1}$-expanding alphabet, and let $p$ be an element of CQC-WFF $A_{1}$. The functor $A_{4}$-Cast $p$ yields an element of CQC-WFF $A_{4}$ and is defined by:

(Def. 3) $\quad A_{4}$-Cast $p=p$. 
Let us consider $A_{1}$, let $A_{4}$ be an $A_{1}$-expanding alphabet, and let $x$ be a bound variable of $A_{1}$. The functor $A_{4}$-Cast $x$ yields a bound variable of $A_{4}$ and is defined as follows:

(Def. 4) $\quad A_{4}$-Cast $x=x$.

Let us consider $A_{1}$, let $A_{4}$ be an $A_{1}$-expanding alphabet, let us consider $k$, and let $P$ be a predicate symbol of $k$ and $A_{1}$. The functor $A_{4}$-Cast $P$ yielding a predicate symbol of $k$ and $A_{4}$ is defined as follows:

(Def. 5) $\quad A_{4}$-Cast $P=P$.

Let us consider $A_{1}$, let $A_{4}$ be an $A_{1}$-expanding alphabet, let us consider $k$, and let $l$ be a CQC-variable list of $k$ and $A_{1}$. The functor $A_{4}$-Cast $l$ yielding a CQC-variable list of $k$ and $A_{4}$ is defined as follows:

(Def. 6) $\quad A_{4}$-Cast $l=l$.

Next we state the proposition

(8) Let given $p, r, x, P, l$ and $A_{4}$ be an $A_{1}$-expanding alphabet. Then $A_{4}$-Cast VERUM $A_{1}=\operatorname{VERUM} A_{4}$ and $A_{4}$-Cast $P[l]=$ $\left(A_{4}\right.$-Cast $\left.P\right)\left[A_{4}\right.$-Cast $\left.l\right]$ and $A_{4}$-Cast $\neg p=\neg\left(A_{4}\right.$-Cast $\left.p\right)$ and $A_{4}$-Cast $(p \wedge$ $r)=\left(A_{4}\right.$-Cast $\left.p\right) \wedge\left(A_{4}\right.$-Cast $\left.r\right)$ and $A_{4}$-Cast $\forall_{x} p=\forall_{A_{4} \text {-Cast } x}\left(A_{4}\right.$-Cast $\left.p\right)$.

\section{Downward Transfer of Consistency and Satisfiability}

The following propositions are true:

(9) Suppose $J_{1}=J_{2}$ †the predicate symbols of $A_{1}$ and $v_{1}=v_{2}\lceil$ the bound variables of $A_{1}$. Then $J_{2}={ }_{v_{2}} A_{4}$-Cast $r$ if and only if $J_{1}={ }_{v_{1}} r$.

(10) Let $A_{4}$ be an $A_{1}$-expanding alphabet and $T_{1}$ be a subset of CQC-WFF $A_{4}$. Suppose $P_{1} \subseteq T_{1}$. Let $A_{2}$ be a non empty set, $J_{2}$ be an interpretation of $A_{4}$ and $A_{2}$, and $v_{2}$ be an element of the valuations in $A_{4}$ and $A_{2}$. If $J_{2} \models_{v_{2}} T_{1}$, then there exist $A, J, v$ such that $J \models_{v} P_{1}$.

(11) Let $f$ be a finite sequence of elements of CQC-WFF $A_{4}$ and $g$ be a finite sequence of elements of CQC-WFF $A_{1}$. If $f=g$, then $\operatorname{Ant}(f)=\operatorname{Ant}(g)$ and $\operatorname{Suc}(f)=\operatorname{Suc}(g)$.

(12) For every $p$ holds the still not bound in $p=$ the still not bound in $A_{4}$-Cast $p$.

(13) Let $p_{2}$ be an element of CQC-WFF $A_{4}, S$ be a substitution of $A_{1}$, $S_{2}$ be a substitution of $A_{4}, x_{2}$ be a bound variable of $A_{4}$, and given $x, p$. If $p=p_{2}$ and $S=S_{2}$ and $x=x_{2}$, then $\operatorname{RestrictSub}(x, p, S)=$ RestrictSub $\left(x_{2}, p_{2}, S_{2}\right)$.

(14) Let $p_{2}$ be an element of CQC-WFF $A_{4}, S$ be a finite substitution of $A_{1}$, $S_{2}$ be a finite substitution of $A_{4}$, and given $p$. If $S=S_{2}$ and $p=p_{2}$, then $\operatorname{up} \operatorname{Var}(S, p)=u p \operatorname{Var}\left(S_{2}, p_{2}\right)$. 
(15) Let $p_{2}$ be an element of CQC-WFF $A_{4}, S$ be a substitution of $A_{1}, S_{2}$ be a substitution of $A_{4}, x_{2}$ be a bound variable of $A_{4}$, and given $x, p$. If $p=p_{2}$ and $S=S_{2}$ and $x=x_{2}$, then $\operatorname{ExpandSub}\left(x, p, \operatorname{RestrictSub}\left(x, \forall_{x} p, S\right)\right)=$ $\operatorname{Expand} \operatorname{Sub}\left(x_{2}, p_{2}, \operatorname{RestrictSub}\left(x_{2}, \forall_{x_{2}} p_{2}, S_{2}\right)\right)$.

(16) Let $Z$ be an element of CQC-Sub-WFF $A_{1}$ and $Z_{2}$ be an element of CQC-Sub-WFF $A_{4}$. Suppose $Z_{1}$ is universal and $\left(Z_{2}\right)_{1}$ is universal and $\operatorname{Bound}\left(Z_{\mathbf{1}}\right)=\operatorname{Bound}\left(\left(Z_{2}\right)_{1}\right)$ and $\operatorname{Scope}\left(Z_{\mathbf{1}}\right)=\operatorname{Scope}\left(\left(Z_{2}\right)_{1}\right)$ and $Z=Z_{2}$. Then S-Bound $\left({ }^{@} Z\right)=\mathrm{S}-\mathrm{B}$ ound $\left({ }^{@} Z_{2}\right)$.

(17) Let $p_{2}$ be an element of CQC-WFF $A_{4}, x_{2}, y_{2}$ be bound variables of $A_{4}$, and given $p, x, y$. If $p=p_{2}$ and $x=x_{2}$ and $y=y_{2}$, then $p(x, y)=$ $p_{2}\left(x_{2}, y_{2}\right)$.

(18) For every consistent subset $P_{1}$ of CQC-WFF $A_{4}$ such that $P_{1}$ is a subset of CQC-WFF $A_{1}$ holds $P_{1}$ is $A_{1}$-consistent.

\section{Upward Transfer of Consistency and Satisfiability}

Next we state two propositions:

(19) For every $p$ there exists a countable alphabet $A_{3}$ such that $p$ is an element of CQC-WFF $A_{3}$ and $A_{1}$ is $A_{3}$-expanding.

(20) Let $P_{1}$ be a finite subset of CQC-WFF $A_{1}$. Then there exists a countable alphabet $A_{3}$ such that $P_{1}$ is a finite subset of CQC-WFF $A_{3}$ and $A_{1}$ is $A_{3^{-}}$ expanding.

Let us consider $A_{1}$ and let $P_{1}$ be a finite subset of CQC-WFF $A_{1}$. Note that the still not bound in $P_{1}$ is finite.

Next we state three propositions:

(21) Let $T_{1}$ be a subset of CQC-WFF $A_{4}$. Suppose $P_{1}=T_{1}$. Let given $A$, $J, v$. Suppose $J \models{ }_{v} P_{1}$. Then there exists a non empty set $A_{2}$ and there exists an interpretation $J_{2}$ of $A_{4}$ and $A_{2}$ and there exists an element $v_{2}$ of the valuations in $A_{4}$ and $A_{2}$ such that $J_{2} \models_{v_{2}} T_{1}$.

(22) For every subset $C_{1}$ of CQC-WFF $A_{1}$ such that $C_{1} \subseteq P_{1}$ holds $C_{1}$ is consistent.

$P_{1}$ is $A_{4}$-consistent.

\section{REFERENCES}

[1] Grzegorz Bancerek. Cardinal numbers. Formalized Mathematics, 1(2):377-382, 1990.

[2] Grzegorz Bancerek. König's theorem. Formalized Mathematics, 1(3):589-593, 1990.

[3] Grzegorz Bancerek. The ordinal numbers. Formalized Mathematics, 1(1):91-96, 1990.

[4] Grzegorz Bancerek and Krzysztof Hryniewiecki. Segments of natural numbers and finite sequences. Formalized Mathematics, 1(1):107-114, 1990.

[5] Patrick Braselmann and Peter Koepke. Coincidence lemma and substitution lemma. Formalized Mathematics, 13(1):17-26, 2005. 
[6] Patrick Braselmann and Peter Koepke. Equivalences of inconsistency and Henkin models. Formalized Mathematics, 13(1):45-48, 2005.

[7] Patrick Braselmann and Peter Koepke. Gödel's completeness theorem. Formalized Mathematics, 13(1):49-53, 2005.

[8] Patrick Braselmann and Peter Koepke. A sequent calculus for first-order logic. Formalized Mathematics, 13(1):33-39, 2005.

[9] Patrick Braselmann and Peter Koepke. Substitution in first-order formulas: Elementary properties. Formalized Mathematics, 13(1):5-15, 2005.

[10] Patrick Braselmann and Peter Koepke. Substitution in first-order formulas. Part II. The construction of first-order formulas. Formalized Mathematics, 13(1):27-32, 2005.

[11] Czesław Byliński. A classical first order language. Formalized Mathematics, 1(4):669-676, 1990.

[12] Czesław Byliński. Functions and their basic properties. Formalized Mathematics, 1(1):5565, 1990.

[13] Czesław Byliński. Functions from a set to a set. Formalized Mathematics, 1(1):153-164, 1990.

[14] Czesław Byliński. Some basic properties of sets. Formalized Mathematics, 1(1):47-53, 1990.

[15] Agata Darmochwał. Finite sets. Formalized Mathematics, 1(1):165-167, 1990.

[16] Agata Darmochwał. A first-order predicate calculus. Formalized Mathematics, 1(4):689$695,1990$.

[17] Kurt Gödel. Die Vollständigkeit der Axiome des logischen Funktionenkalküls. Monatshefte für Mathematik und Physik 37, 1930.

[18] W. Thomas H.-D. Ebbinghaus, J. Flum. Einführung in die Mathematische Logik. Springer-Verlag, Berlin Heidelberg, 2007.

[19] Piotr Rudnicki and Andrzej Trybulec. A first order language. Formalized Mathematics, 1(2):303-311, 1990.

[20] Zinaida Trybulec. Properties of subsets. Formalized Mathematics, 1(1):67-71, 1990

[21] Edmund Woronowicz. Interpretation and satisfiability in the first order logic. Formalized Mathematics, 1(4):739-743, 1990.

[22] Edmund Woronowicz. Many argument relations. Formalized Mathematics, 1(4):733-737, 1990.

[23] Edmund Woronowicz. Relations and their basic properties. Formalized Mathematics, 1(1):73-83, 1990.

[24] Edmund Woronowicz. Relations defined on sets. Formalized Mathematics, 1(1):181-186, 1990.

Received May 7, 2012 